\title{
Research Paper: Effect of Hemipelvectomy Amputation on Kinematics and Muscle Force Generation of Lower Limb While Walking
}

\author{
Keyvan Sharifmoradi' ${ }^{1}$ *Mostafa Kamali², Mohammad Taghi Karimi ${ }^{2}$
}

1. Department of Physical Education, Faculty of Humanities, University of Kashan, Kashan, Iran.

2. Department of Orthotics and Prosthetics, Faculty of Rehabilitation, Isfahan University of Medical Sciences, Isfahan, Iran.

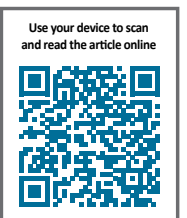

Citation: Sharifmoradi K, Kamali M, Karimi MT. [Effect of Hemipelvectomy Amputation on Kinematics and Muscle Force Generation of Lower Limb While Walking (Persian)]. Archives of Rehabilitation. 2017; 18(2):122-131. http://dx.doi. org/10.21859/jrehab-1802120

http://dx.doi.org/10.21859/jrehab-1802120

Received: 04 Nov. 2016 Accepted: 17 Mar. 2017

Keywords: Hemipelvectomy amputation, Muscle force, Kinematics, Gait

\section{ABSTRACT}

Objective Hemipelvectomy amputation is a surgical procedure in which the lower limb and a portion of pelvic are removed. There are a few studies on the performance of this group of patients while walking. The aim of this paper was to evaluate the effect of hemipelvectomy amputation on kinematics and muscle force generation of the lower limb while walking with Canadian prosthesis.

Materials \& Methods A subject who underwent hemipelvectomy amputation on his left side and whose mass, height, and age were $75 \mathrm{~kg}, 1.75 \mathrm{~m}$, and 39 years, respectively, was involved in this study. Qualisys motion analysis system with seven cameras and force-plate system were used to record marker tracking and ground reaction forces. Twenty reflective markers were attached to the subject's body. As the subject walked, the data was recorded. The mean of five trials was used for statistical computing. The data was collected with the frequency of $120 \mathrm{~Hz}$ and filtered with $10 \mathrm{~Hz}$ low-pass filter. Muscloskeletal modeling was conducted by Visual 3D and OpenSim software. All data were analyzed using the SPSS 19 software at $\alpha=0.05$.

Results There were significant differences between knee and ankle joint kinematic pattern at Loading, Mid-stance, Terminal stance, and Pre-swing phases of gait $(P<0.05)$.

In weight acceptance phase and mid-stance phase, ankle plantar flexion and dorsi-flexion range of motion, respectively, were significantly lower in the patient compared to a healthy subject $(P=0.00)$. At the end of the stance phase, ankle range of motion was significantly different in the patient compared to the healthy subject $(P=0.00)$. In the pre-swing phase, ankle plantar flexion was 11.5 degrees greater than that of the healthy subject. In mid-swing phase, patients showed more ankle dorsi-flexion compared to the healthy subject. In weight acceptance phase, knee flexion of patient $(P=0.00)$ was significantly greater than that of the healthy subject, and in mid-stance phase, knee extension of patient was significantly greater $(P=0.04)$. In pre-swing phase $(P=0.00)$ and initial swing $(P=0.02)$, there were significant differences between knee flexion of patients and healthy subjects. The pattern of hip range of motion during gait cycle was the same for the patient and the healthy subject $(P>0.05)$. Force generation of the hip abductor, hip extensor, and knee extensor along with tibialis anterior and posterior of the patients in the sound leg were significantly more than that of the normal subject $(\mathrm{P}<0.05)$.

Conclusion The kinematics pattern of the patient's lower limb during gait is different. Kinematic changes are associated with a significant increase in lower limb muscle generation that can have a degenerative effect on the knee joint. So the importance of this subject should be considered by rehabilitation experts.

\section{*Corresponding Author: \\ Mostafa Kamali, MSc.}

Address: Department of Orthotics and Prosthetics, Faculty of Rehabilitation, Isfahan University of Medical Sciences, Isfahan, Iran.

Tel: +98 (913) 3505258

E-Mail: mostafa_kamali@rehab.mui.ac.ir 


\title{
اثر قطع عضو هميبلويكتومى بر كينماتيكى و نيروى توليدى عضلات اندام تحتانى حين راه رفتن با

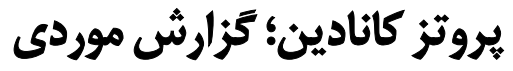

\author{
كيوان شريف مرادى'، "مصطفى كمالى'، محمدتقى كريمى' \\ ا - كروه علوم ورزشى، دانشكده علوم انسانى، دانشكاه كاشان، كاشان، ايران.

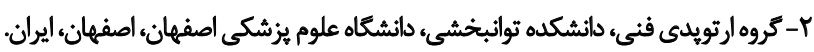

\begin{abstract}
حكSe

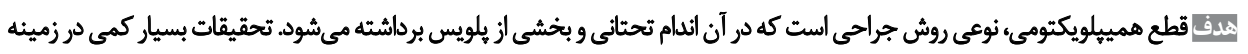

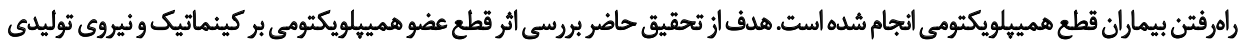

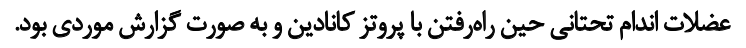

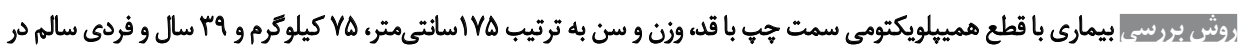

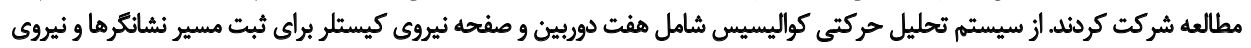

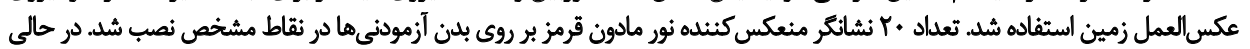

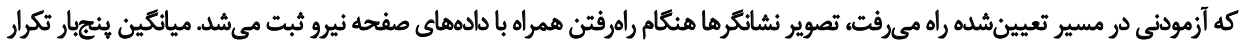

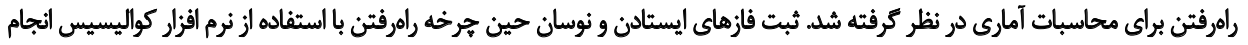

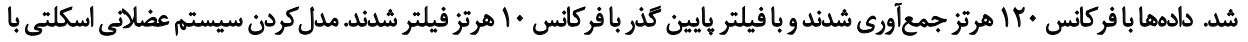

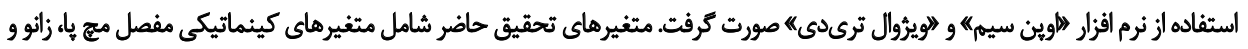

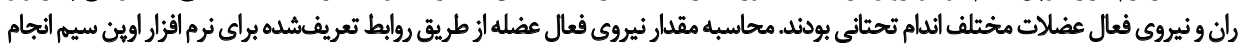

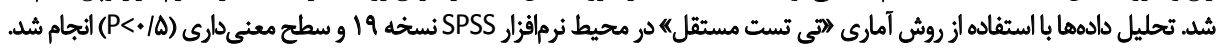

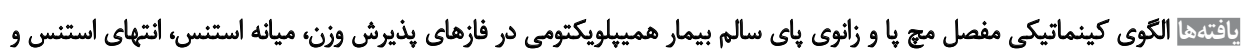

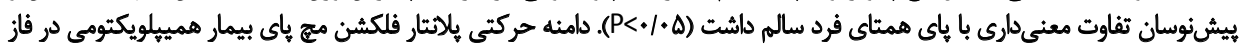

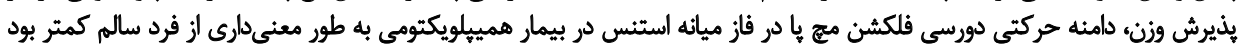

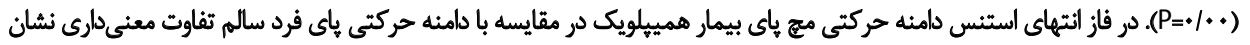

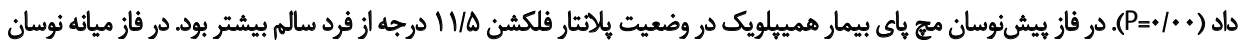

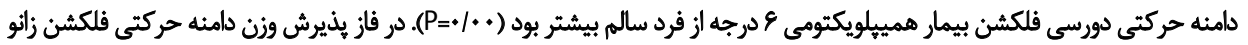

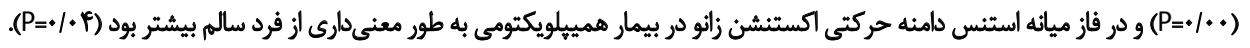

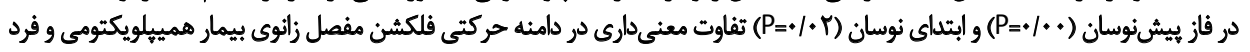

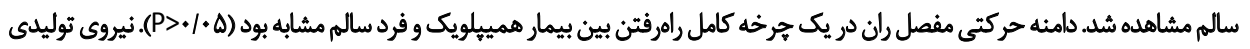

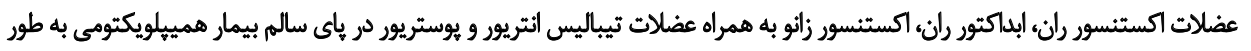

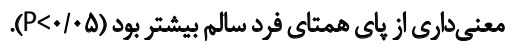

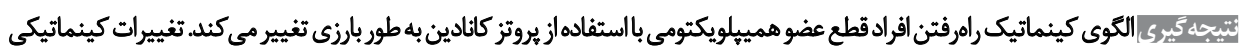

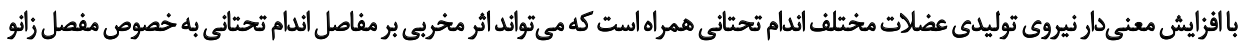

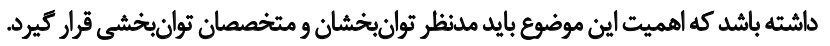

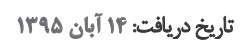

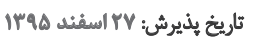

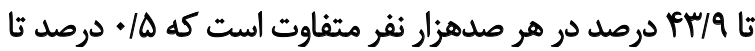
datero

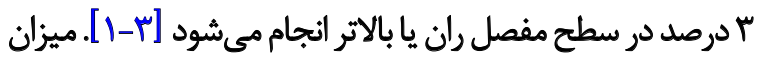

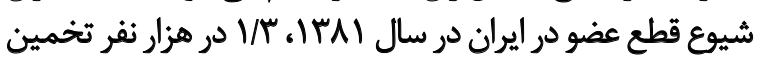

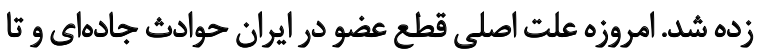
قطع عضوهاى اندام تحتانى در نتيجه تروما، بيمارىهاى

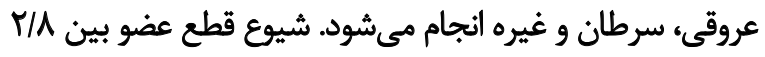
드.

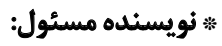 مصطفي كمالي}

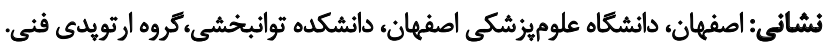
تلفن: رايانامه: mostafa_kamali@rehab.mui.ac.ir 


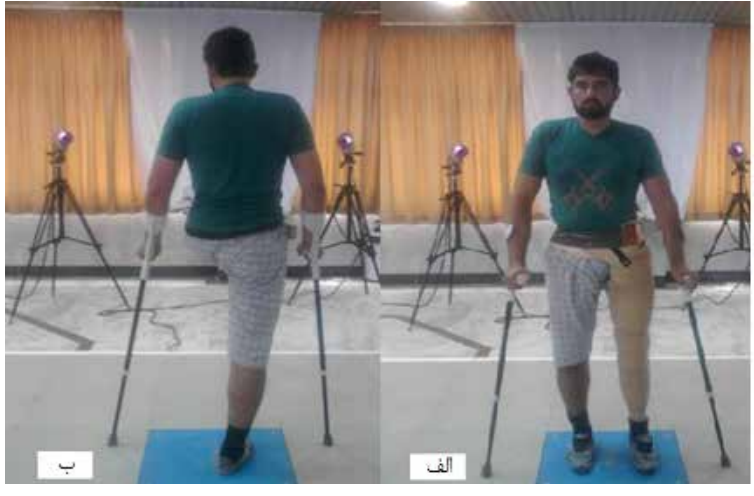

توانبخننى

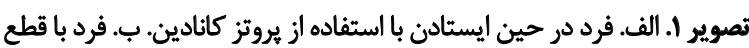
عضو همييلويك بدون يروتز

بر طبق منابع در دسترس، تحقيقات انجامشده در زميئه

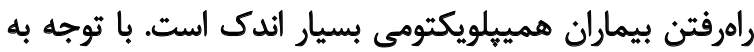

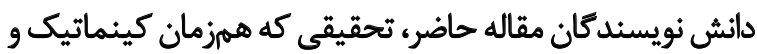

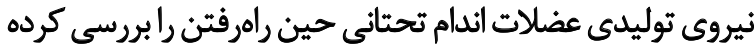

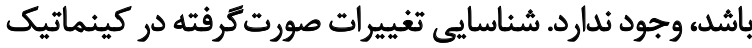

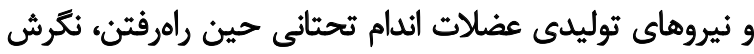

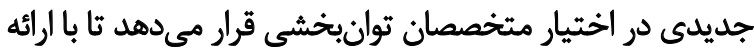

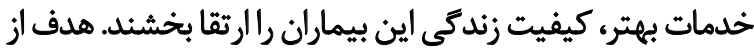

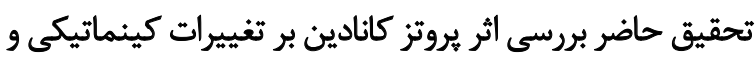

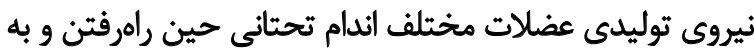

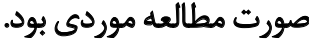

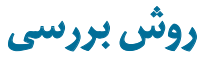

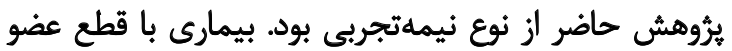

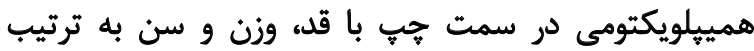

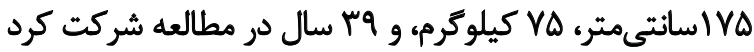

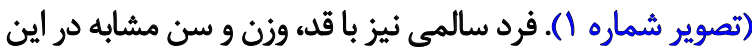

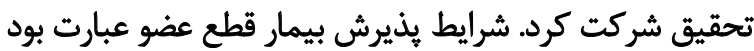

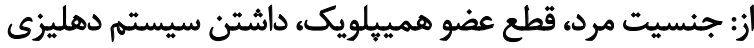

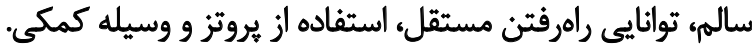

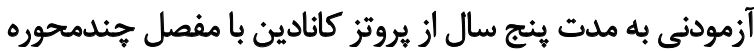

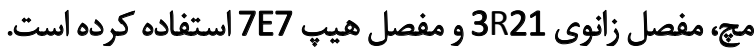

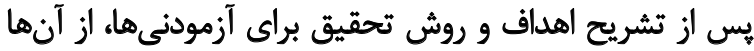
رضايتنامه كتبى براي شركت در اين يثوهش تركيت ترفته شد.

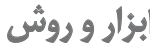

براى اندازمكيرى متغيرهاي كينماتيكى راهرفتن از سيستم

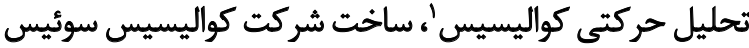

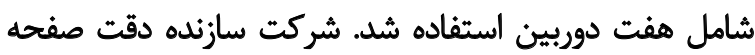

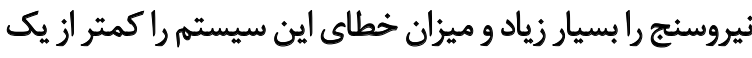

حدودى حوادث در محيط كار است. بنابراين برنامهريزى در جهت

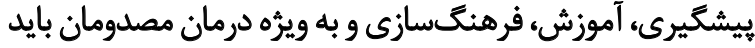

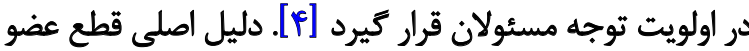

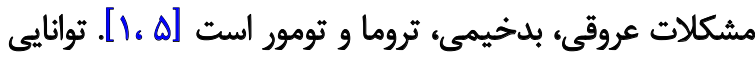

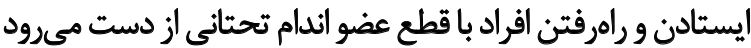

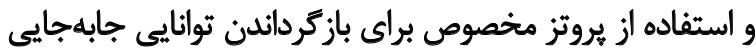

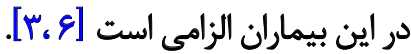

قطع عضو همييلويكتومى نوعى روش جراحى است كه كل بل بردي

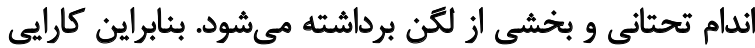

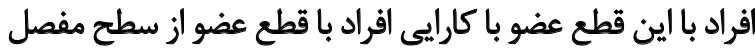

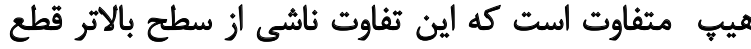

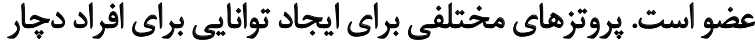

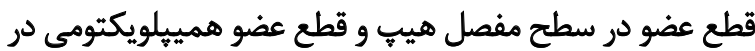

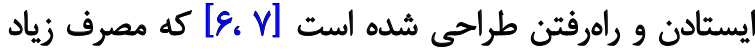

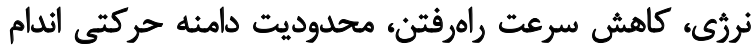

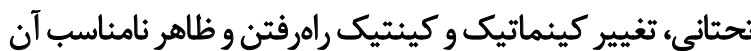

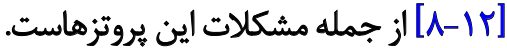

مطالعات درباره راهرفتن افراد دجار قطع عضو همييلويك بسيار

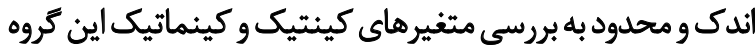

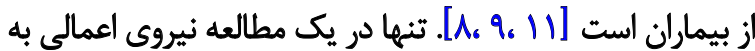

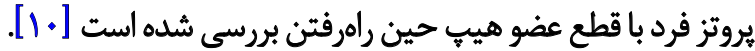

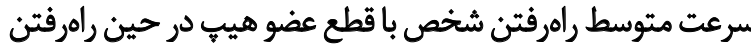

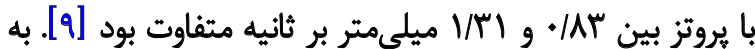

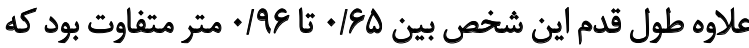

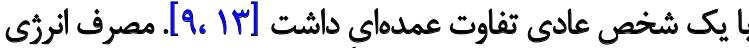

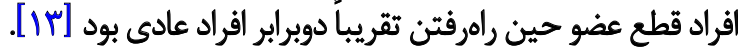

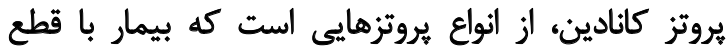

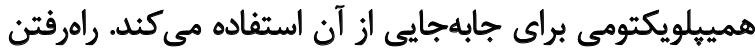

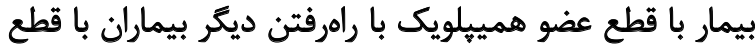

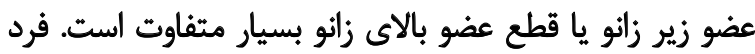

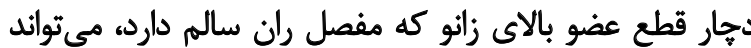

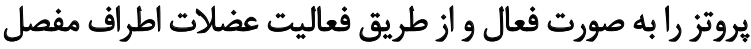

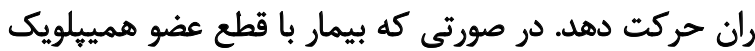

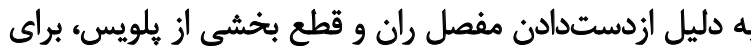

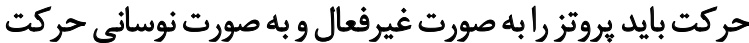

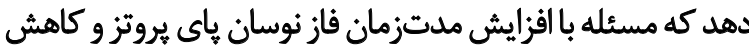

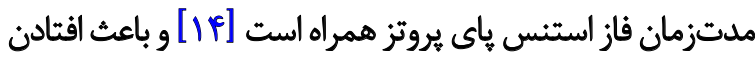

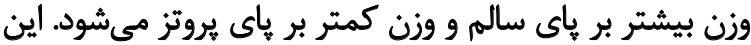

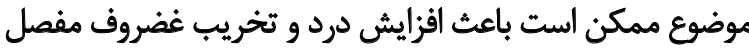

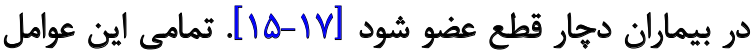

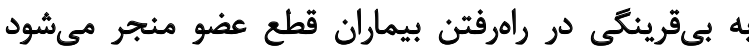

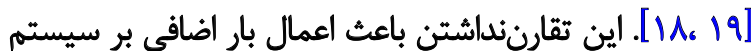

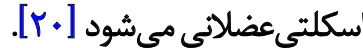




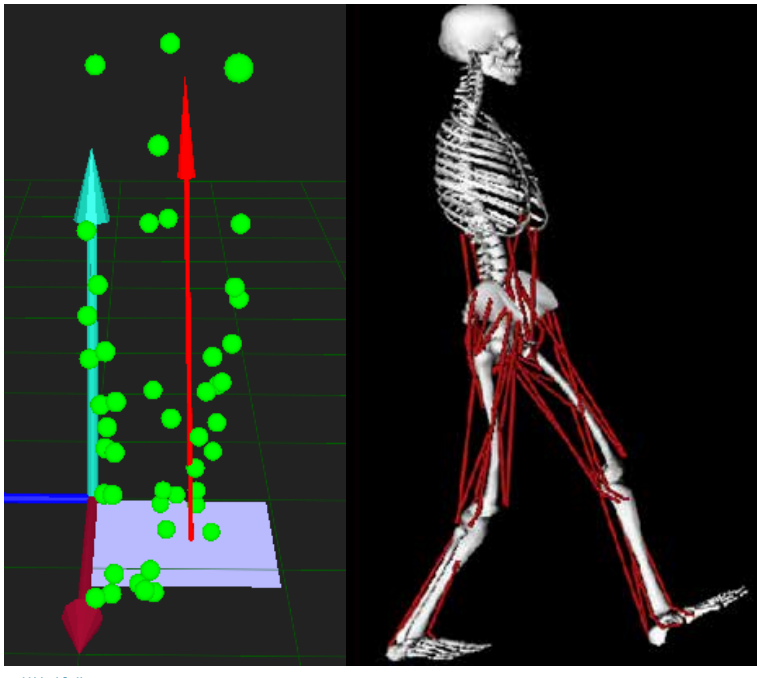

توانبخننى

تصوير Y. محل قراركيرى نشانكرها در نرمافزار كواليسيس و مدل عضلانى

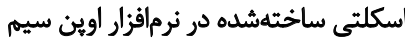

قدامي راست، عرض مج يا سمت راست بدن ثبت شد. سيس

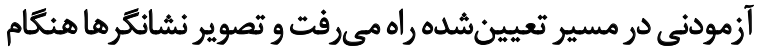

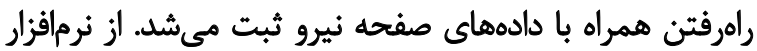

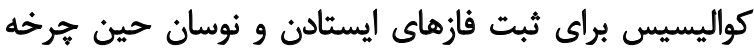
راهرتن استفاده شد.

$$
\text { مرالجال إمرا }
$$

يس از تنظيم دوربينها و نصب نشانكرها، آزمودنى بدون

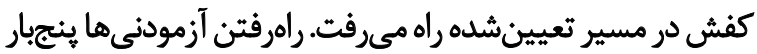

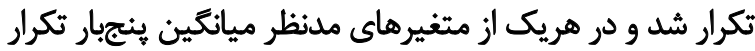

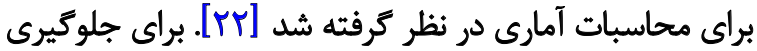

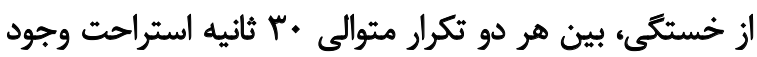

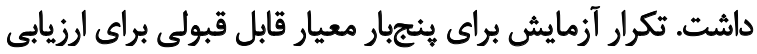

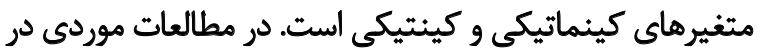

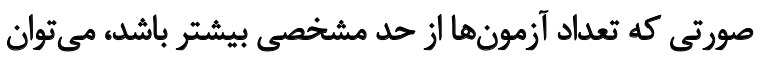

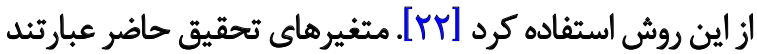

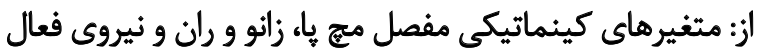

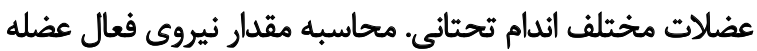

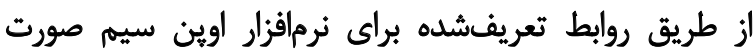

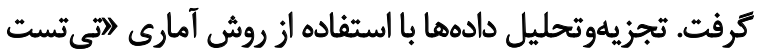

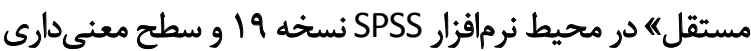

$$
\text { (P<-1Q) }
$$

\section{يافتهها}

دامنه حركتى مفصل ميج يا در يك جرخه كامل مامل راهرفتن در

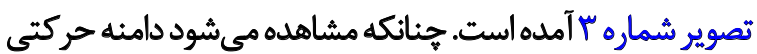

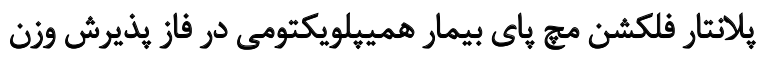

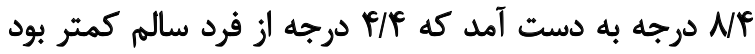

درصد اعلام كرده است [r]].

دوربينها در دو سمت يك مسير ييادهرو و به فاصله جهار

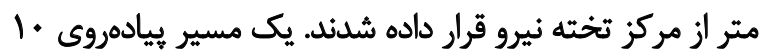

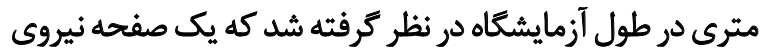

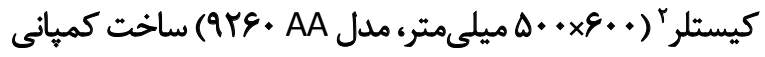

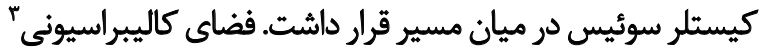

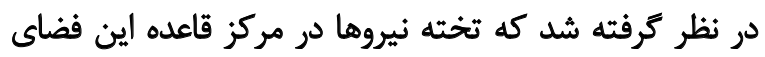

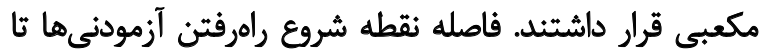
تخته نيرو ينج متر بود.

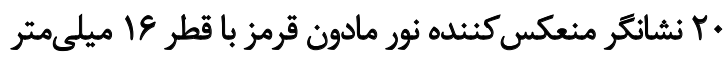

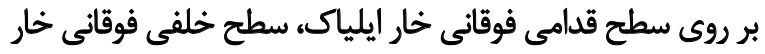

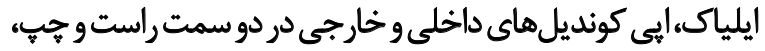

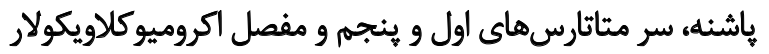

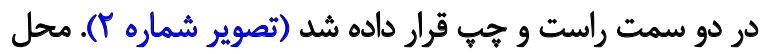

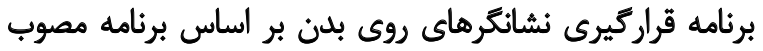

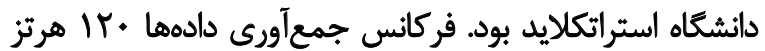

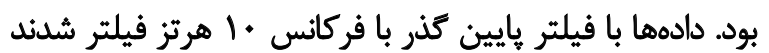

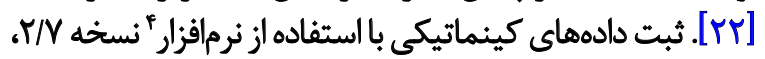

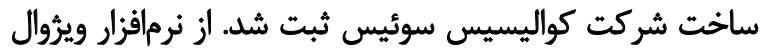

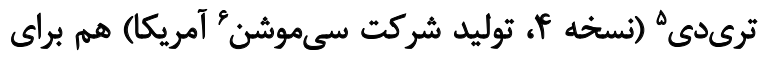

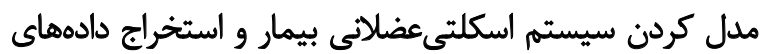

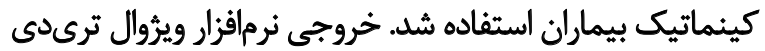

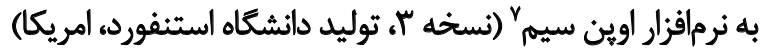

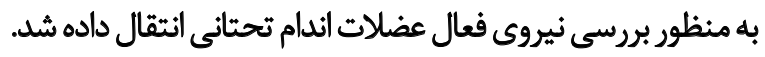

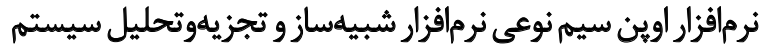

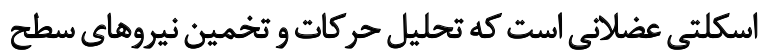

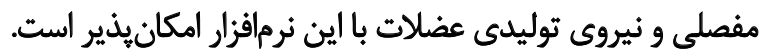

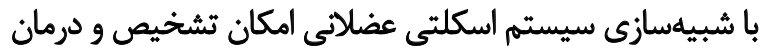

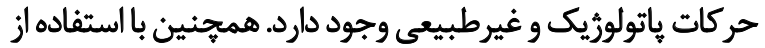

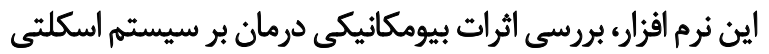

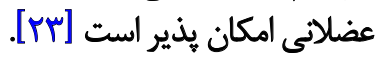
يس از كاليبره كردن دوربينها و صفحه نيرو ابتدا دادههاى

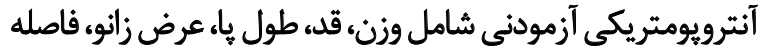

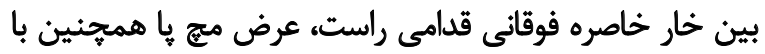

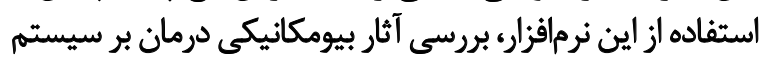

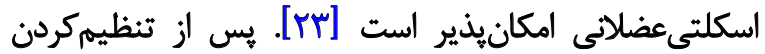

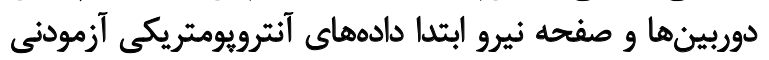

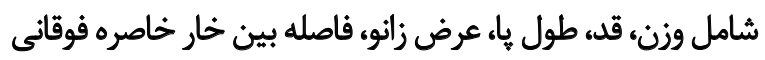

\section{2. kistler}

"ا. فضاى مكعبى شكل فرضى داخل محيط آزمايشكاه كه حركت آزمونى در آنجا ثبت مىشود. 4. Qualysis Track Manager

5. Visual 3D

6. C-motion

7. Open SIMM 


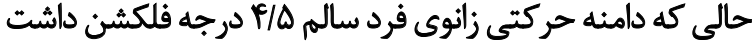

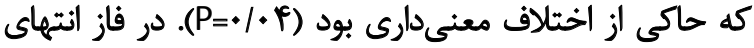

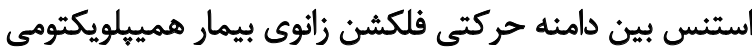

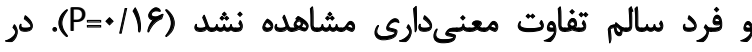

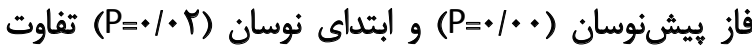

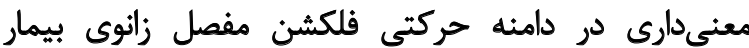
همييلويكتومى و فرد سالم مشاهده شد.

تصوير شماره ه دامنه حركتى مفصل ران در يك جرخد

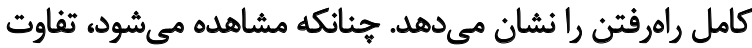

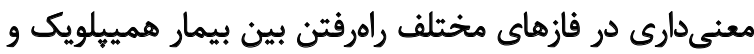

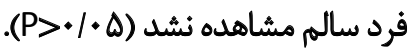

جدول شماره ا نيروى فعال توليدشده توسط عضلات اندام

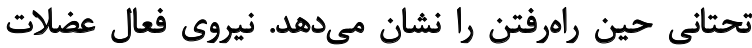

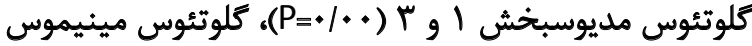

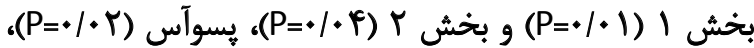

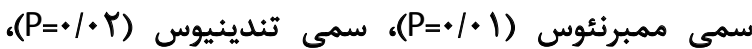

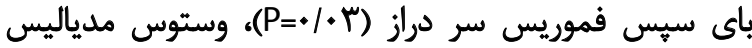

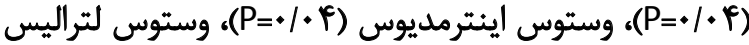

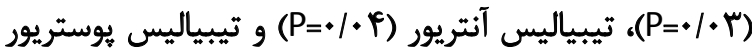

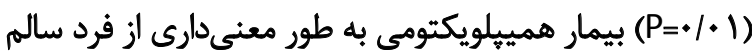

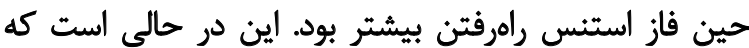

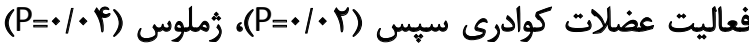

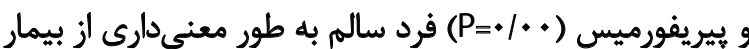

همييلويك بيشتر بود.

بحث

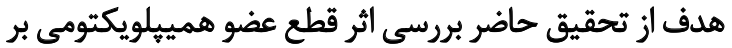

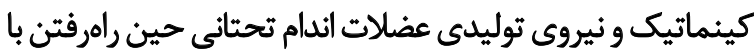

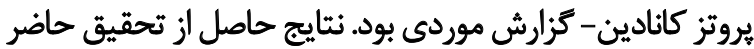

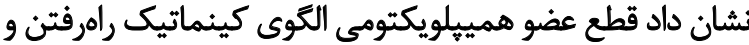

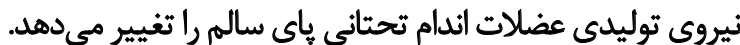

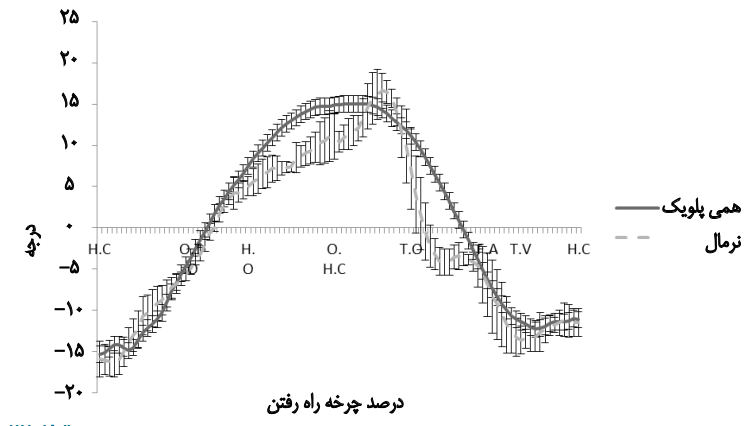

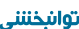

تصوير ه. دامنه حركتى مفصل زانوى ياى راست فرد همييلويك در مقايسه

با قرد سالم (درجه)

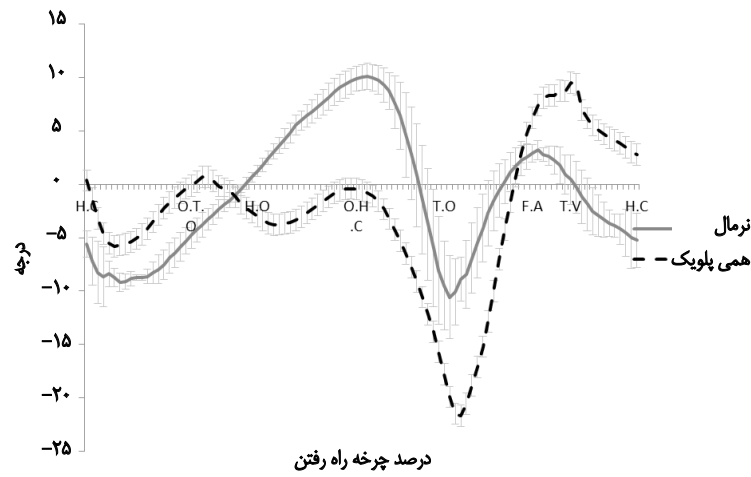

توانبخننى

تصوير ب. دامنه حركتى ميج باي راست فرد همييلويك در مقايسه با فرد

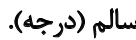

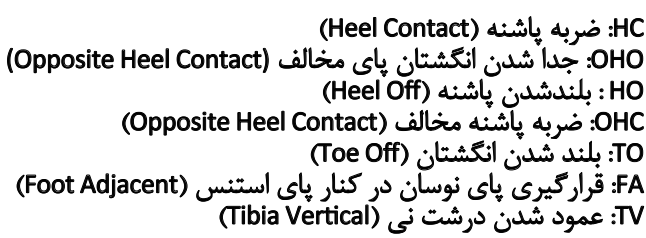

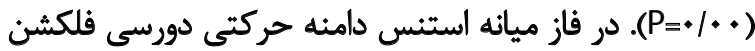

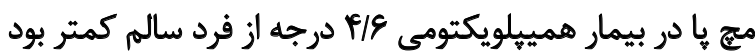

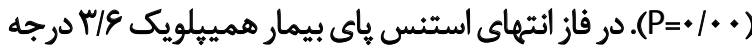

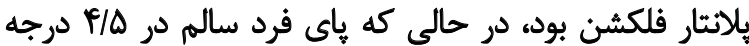

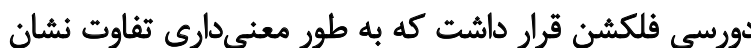

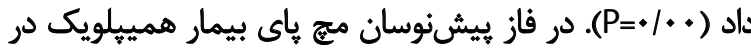

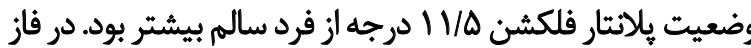

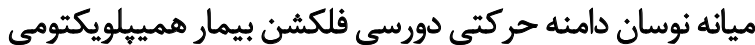

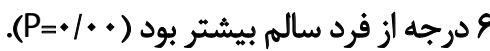

تصوير شماره f دامنه حركتى مفصل زانو در جرخد

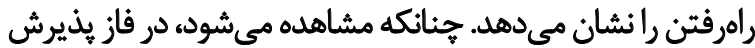

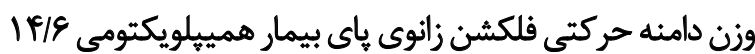

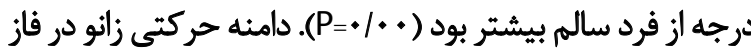
ميانه استنس در بيمار همييلويكتومى در اكستنشن كامل بود، در برد

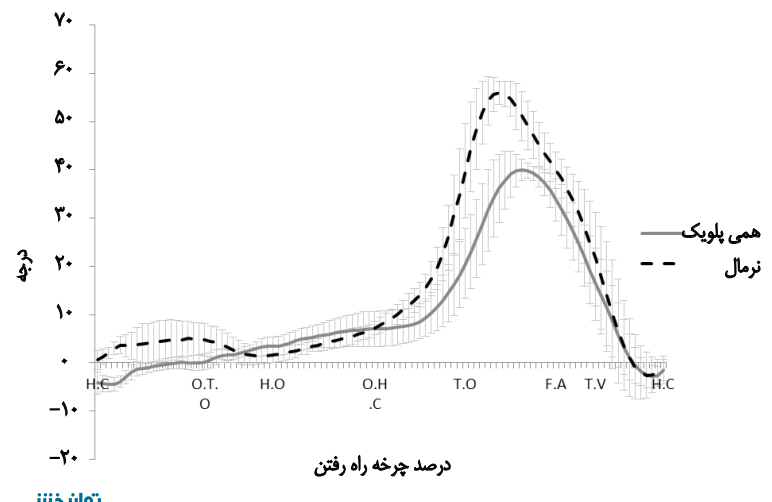

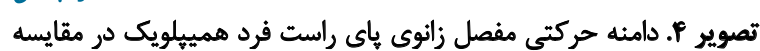

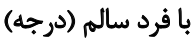


جدول ا. نيروى فعال توليدشده توسط عضلات اندام تحتانى حين راهرفتن

\begin{tabular}{|c|c|c|c|}
\hline تفاوت معنى دار & ثاي راست فرد سالم & هاى رأست بيمار همييلويك & نيروى عضله (نيوتن) \\
\hline$\%$ & $\Delta F / V \pm \Delta F / \lambda$ & $A T \cdot / A \pm T H / T$ & كلوتئوس مديوس 1 \\
\hline - & rquA \pm VII & $r \Delta V / A \pm r Y / P$ & كلوتئوس مديوس r \\
\hline $1.0^{\circ}$ & $\Delta A 1 / T \pm N Q / 8$ & $R r r / q \pm 1 r / q$ & كلوتثموس هديوس r \\
\hline.$\%$ & $\Lambda \backslash / \backslash \pm P Y / F$ & $r W \cdot \pm 9 F / A$ & كلوتثوس مينيموس ا \\
\hline $.1+4$ & $9 Q / T \pm T \& / N$ & $r \cdot . / Y^{\prime} \pm A T / q$ & كلوتثوس مينيموس r \\
\hline.$/ \pi$ & $\mid \Delta \Delta / \Psi \pm \varepsilon \cdot N$ & $11 V / q \pm r+18$ & كلوتثوس مينيموس ب \\
\hline.$/ \Delta r$ & $r \Delta / A \pm Y \& / q$ & $r E Y / Y \pm N$ & 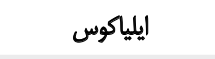 \\
\hline 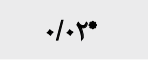 & $19 \Delta / r \pm \wedge 9 / Y$ & $r V q / Y \pm P V /$ & بسوأس \\
\hline.$/ \%$ & $\Delta F / * \pm F \cdot / \Delta$ & $\mid M T / I \pm F N$ & سمى تندينيوس \\
\hline $.1 \% 1^{*}$ & $r V q / V \pm 101 / 8$ & RTNQะR/Y & سمى ممبرائؤوس \\
\hline.$\%$ & $r \mid \& / \Lambda \pm q / / F$ & $M T / T \pm T H N$ & بائ سيس (سر بلند) \\
\hline .1 & $r A V / P \pm I P Y / Q$ & PAT/NIINVI. & باى سيس (سر كوتاه) \\
\hline.$/ M$ & $1 \cdot r / 8 \pm r Q / Y$ & $\mathscr{Q S / \& \pm \Delta Y / q}$ & الداكتور لانكوس \\
\hline .1 .9 & $\gamma+/ T \pm r y / r$ & $r V / q \pm r r / q$ & اداكتور برويس \\
\hline.$/ 4$ & $r \cdot / \cdot \pm 1 Y / \Delta$ & $W \mid \pm r \cdot / r$ & اداكتور مكنوس ا \\
\hline .110 & 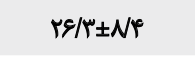 & $\| f / \Delta \pm \mid f / \pi$ & اداكتور مكنوس Y \\
\hline $.19 V$ & $V \& / T \pm T \Delta / T$ & $8 \Delta / Y \pm P T / A$ & اداكتور مكنوس \\
\hline $\mathscr{A A}$ & $\mid r N F \pm N F$ & $\mid f A / q \pm R / q$ & ت تنسور قاسيا لاتا \\
\hline$\circ 1 * 1$ & $r r+/ q \pm \Delta \Delta / 8$ & $\mid q V / T \pm T \Delta / r$ & كلوثتوس ماكزيموس 1 \\
\hline $.19 \Delta$ & $M N Q \pm 1 \otimes / 1$ & $r Q W \backslash \pm \Delta V / Y$ & كلوتثوس ماكزيموس r \\
\hline .1 .8 & $W F \pm F V / \Delta$ & $r \Delta / A \pm Y \& / q$ & كلوتثوس ماكزيموس ب \\
\hline .1 .4 & $\Lambda \sqrt{ } F \pm r \Delta / \Delta$ & rq/A $\pm r \cdot / \Delta$ & كوادروتوس فموريس \\
\hline .10 & $r Q / F \pm Y N A$ & $V / \Delta \pm Y / T$ & زملوس \\
\hline$\% * *$ & $r++/ 1 \pm \Delta V / V$ & $W W F \pm q q$ & ييريفورميس \\
\hline$\%$ & $Y Q D / \cdot \pm Y \& M$ & $1 \varepsilon \cdot / f \pm t \otimes / Y$ & ركتوس فموريس \\
\hline$* 1+e^{+\infty}$ & $q \Psi / 4 \pm \Delta F / \&$ & $19 \% / r \pm V 8 / 0$ & وستّوس مديوس \\
\hline 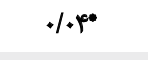 & $1 \cdot V / 1 \pm 8 \Delta / A$ & $\Pi M T / \varepsilon_{ \pm A V I P}$ & وستوس ايثترمديوس \\
\hline $.1 \%$ & $|\Lambda Q / Y \pm| Y \Delta /$. & $P W I \pm I E N A$ & وستوس لتراليس \\
\hline 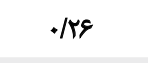 & $999 / r \pm r q 5 / 8$ & $A r q / q \pm \Delta . / r$ & كاستروكنمئوس هديال \\
\hline$\cdot \pi$ & 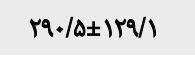 & $r \vee=/ 1 \pm r a / A$ & كاستروكنمثئوس لثرال \\
\hline.$M$ & MTNTEMEV/A & $r|Q \cdot / I \pm r| \Delta / A$ & سولثوس \\
\hline $.1+e^{+\infty}$ & $A \Delta 1 / q \pm 1 \pi T / r$ & $4 \% / r \pm 1 \% / 9$ & تييياليس يوستريور \\
\hline $.1 .1^{*}$ & $\Delta r q / s \pm 11 \Delta / r$ & $V A V / A \pm 1 \cdot \Delta / r$ & تيبياليس انتريور \\
\hline
\end{tabular}


بيماران با قطع عضو اندام تحتانى ^ نشان داد كه كُشتاور هانلاتتار

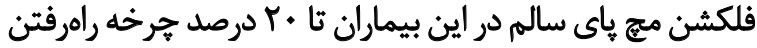

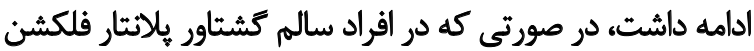

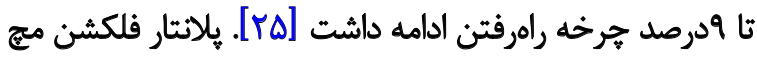

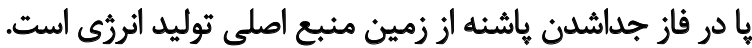

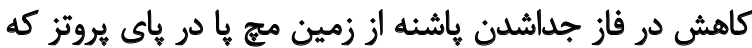

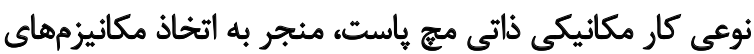

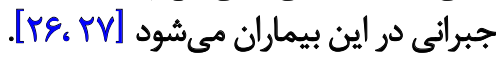
فلكشن زانو در فاز لودينك و حركت به سمت اكستنشن در فاز

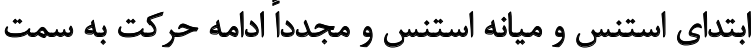

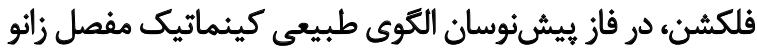

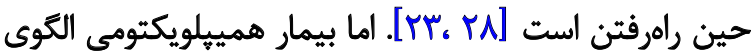

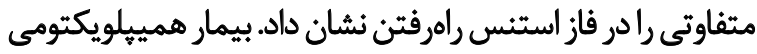

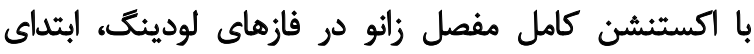

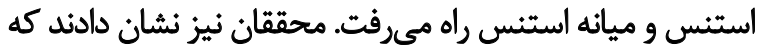

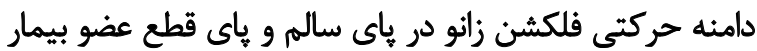

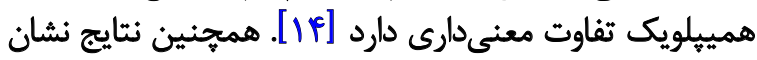

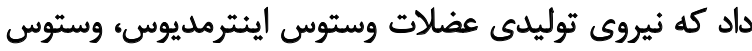

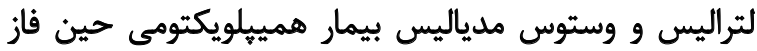

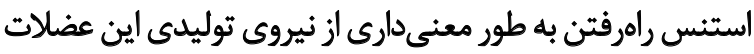
در ياى همسان فرد سالم بيشتر است.

در راهرفتن عادى مقدارى فلكشن در فاز لودينكَ مفصل زانو

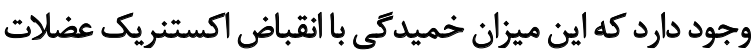

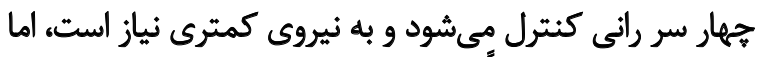

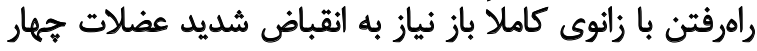

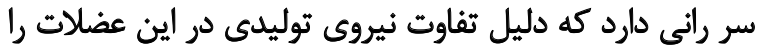

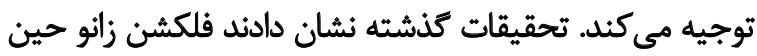

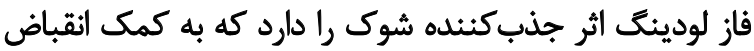

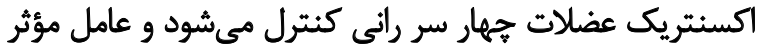

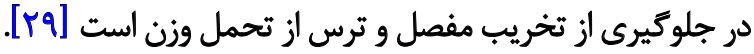

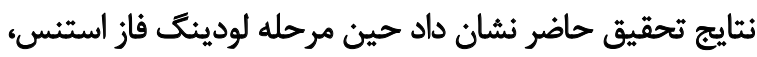

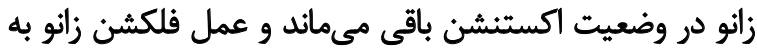

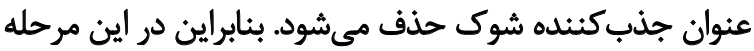

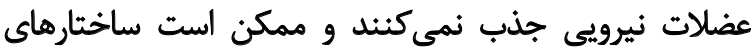

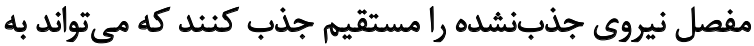
ساختارهاى مفصل زانو آسيب وارد كند.

نيروى توليدى عضلات كلوتئوس مديوس و مينموس هاي

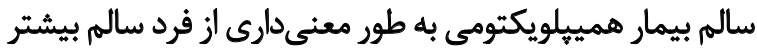

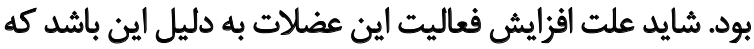

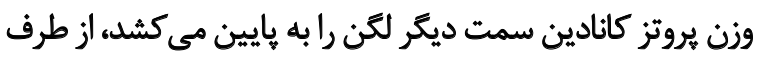

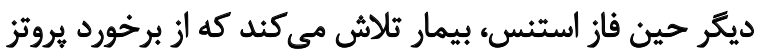

\section{Lower Limb Amputation}

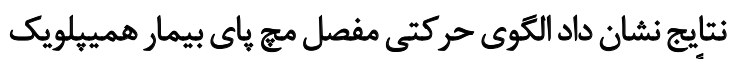

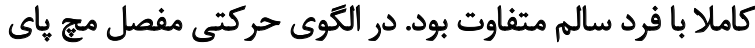

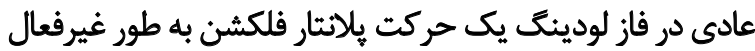

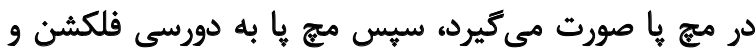

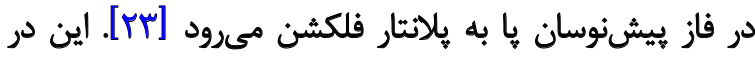

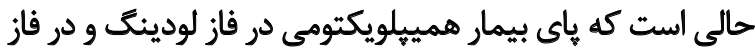

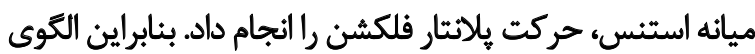

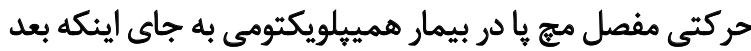

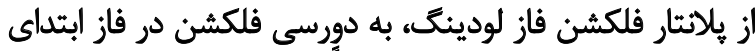

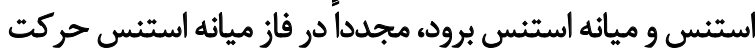

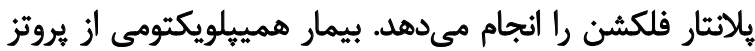

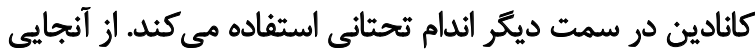

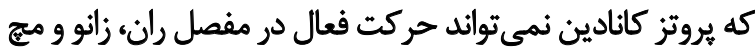

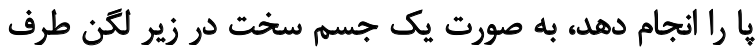

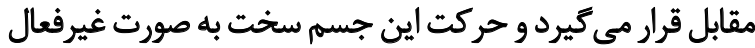

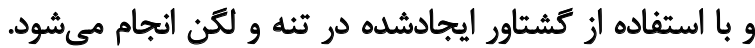

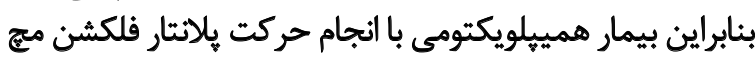

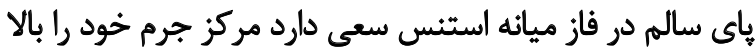

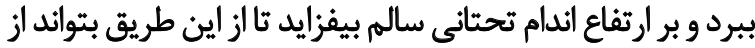
برخورد يروتز كانادين به زمين جلوكيرى كنداند

برخى محققان نشان دادند [rF] كه بيماران با قطع عضو اندام

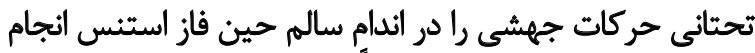

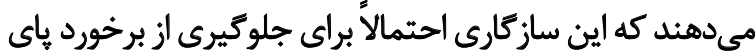

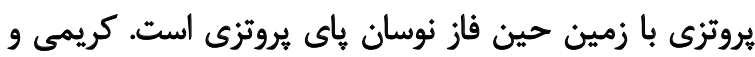

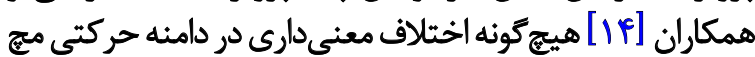

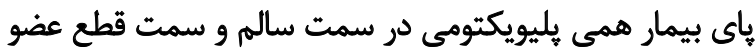

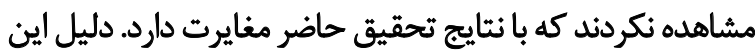

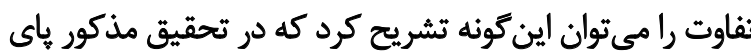

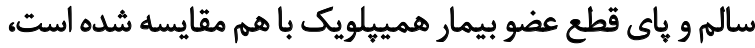

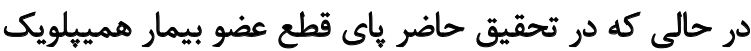

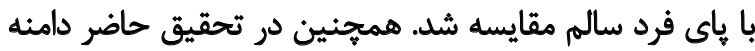

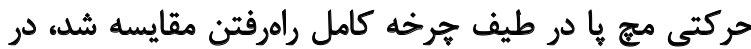

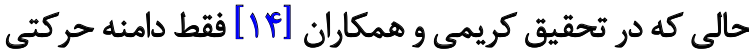

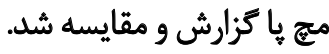

نيروى توليدى عضلات تيباليس انتريور و تيبياليس يوستريور

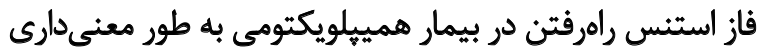

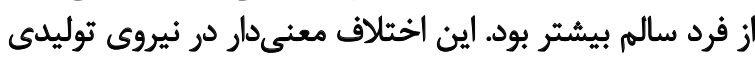

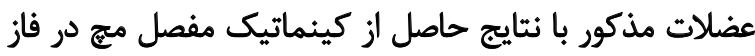

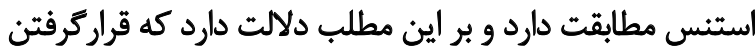

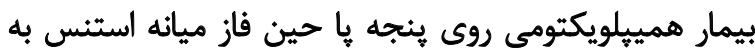

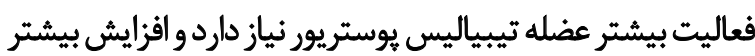

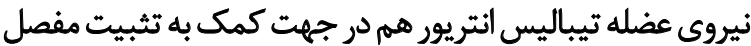

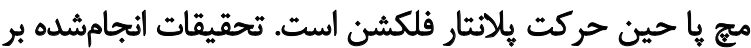


بيشنهاد براى تحقيقات آينده: راهرفتن با زانوى صاف و نداشتن

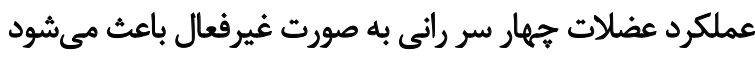

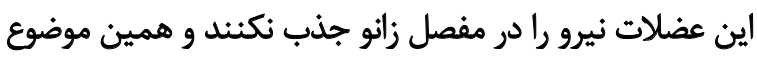

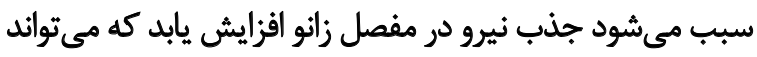

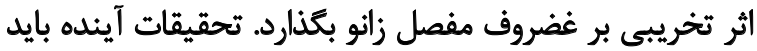

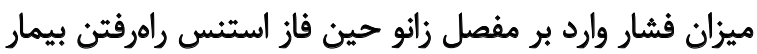
همييلويكتومى رابررسى كند.

$$
\text { تشيكر و قaدرواني }
$$

در هايان از آزمودنى قطع عضو همييلويكتومى به دليل شركت

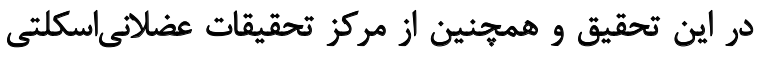

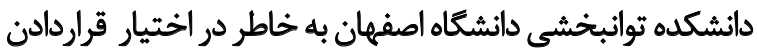

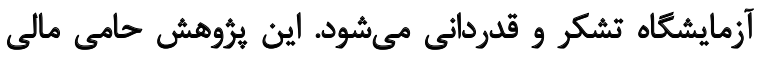

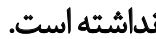

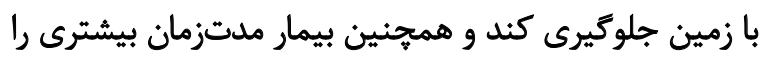

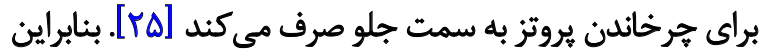

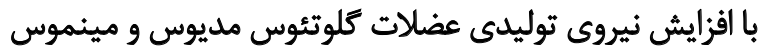

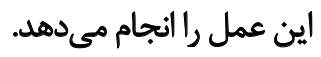

نتايج حاصل از تحقيق حاضر نشان داد نيروى عضلات سمى ني

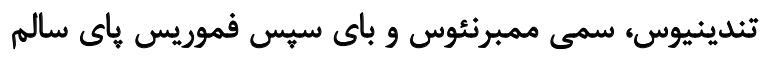

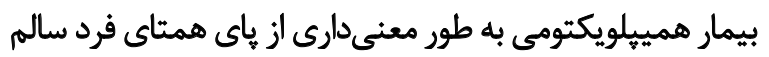

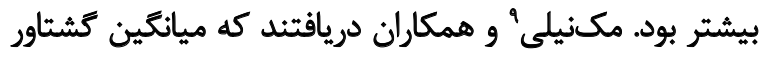

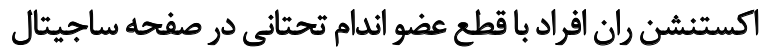

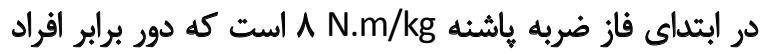

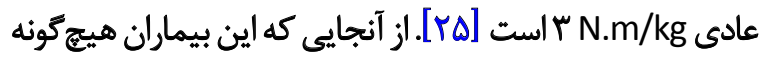

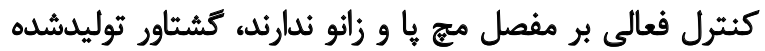

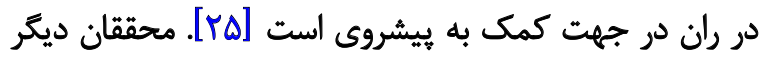

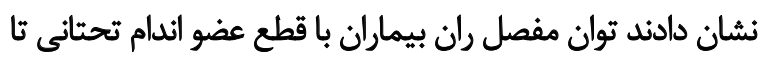

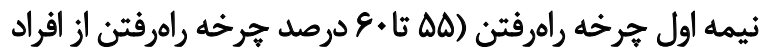

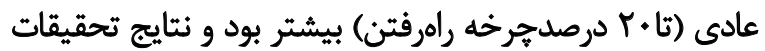

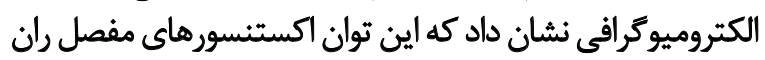

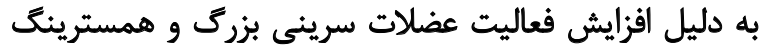

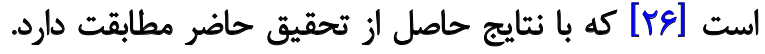

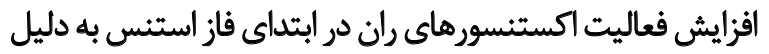

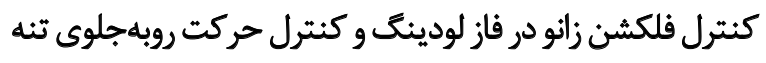
بعد از فاز ضربه بهاشنه است.

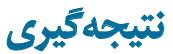

كينماتيكى راهرفتن افراد قطع عضو همييلويكتومى با بتاستفادهاز

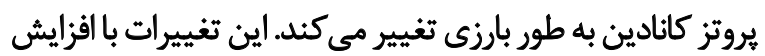

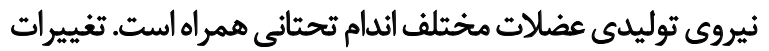

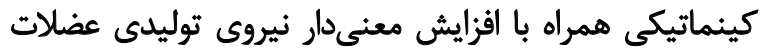

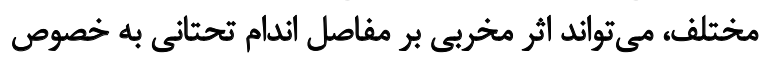

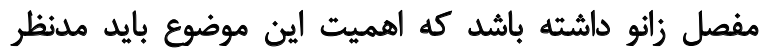

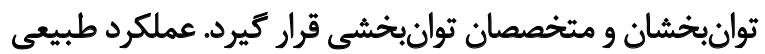

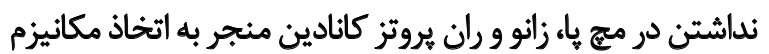
جبرانى عضلات اندام سالم براى راهرفتن شد.

استفاده بيمار همييلويكتومى از عصا حين راهرفتن محدوديت

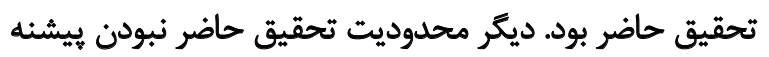

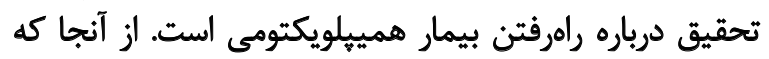

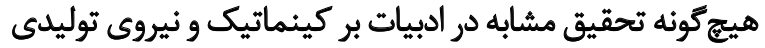

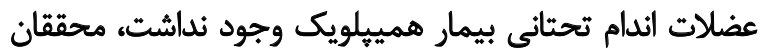

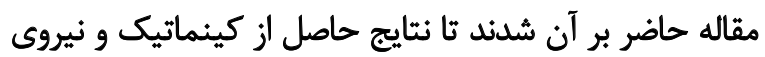

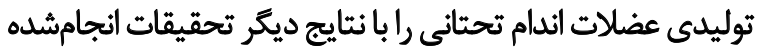
بر قطع عضو اندام تحتانى مقايسه و تفسير كنئد. 


\section{References}

[1] Dillingham TR, Pezzin LE, MacKenzie EJ. Limb amputation and limb deficiency. Southern Medical Journal. 2002; 95(8):875-83. doi: 10.1097/00007611-200295080-00019

[2] Bowker JH, John MW. Atlas of limb prosthetics: Surgical, prosthetic and rehabilitation principles. Missouri: Mosby; 1992.

[3] Smith DG, Michael JW, Bowker JH. Atlas of amputations and limb deficiencies: Surgical, prosthetic, and rehabilitation principles. Rosemont: American Academy of Orthopaedic Surgeons; 2004

[4] Masoudi-Asl I, Nasiri-Pour AA, Faraj-Zadeh F, E'badi M. [Management of work-related injuries leading to amputation and its relation with treatment outcome (Persian)]. Archives of Rehabilitation. 2011; 12(1):34-38.

[5] Unwin N. Epidemiology of lower extremity amputation in centres in Europe, North America and East Asia. British Journal of Surgery. 2000; 87(3):328-37. doi: 10.1046/j.1365-2168.2000.01344.x

[6] Denes Z, Till A .Rehabilitation of patients after hip disarticulation. Archives of Orthopaedic and Trauma Surgery. 1997; 116(8):498-9. doi: 10.1007/s004020050171

[7] Zaffer SM, Braddom RL, Conti A, Goff J, Bokma D. Total hip disarticulation prosthesis with suction socket. American Journal of Physical Medicine \& Rehabilitation. 1999; 78(2):160-2. doi: 10.1097/00002060-199903000-00017

[8] Chin T, Kuroda R, Akisue T, Iguchi T, Kurosaka M. Energy consumption during prosthetic walking and physical fitness in older hip disarticulation amputees. Journal of Rehabilitation Research \& Development; 2012; 49(8):1255-60. doi: 10.1682/ jrrd.2011.04.0067

[9] Ludwigs E, Bellmann M, Schmalz T, Blumentritt S. Biomechanical differences between two exoprosthetic hip joint systems during level walking. Prosthetics and Orthotics International. 2010; 34(4):449-60. doi: 10.3109/03093646.2010.499551

[10] Nietert M, Englisch N, Kreil P, Alba-Lopez G. Loads in hip disarticulation prostheses during normal daily use. Prosthetics and Orthotics International. 1998; 22(3):199-215. doi: $10.3109 / 03093649809164485$

[11] Schnall BL, Baum BS, Andrews AM. Gait characteristics of a soldier with a traumatic hip disarticulation. Physical Therapy. 2008; 88(12):1568-77. doi: 10.2522/ptj.20070337

[12] Yari P, Dijkstra PU, Geertzen JH. Functional outcome of hip disarticulation and hemipelvectomy: A cross-sectional national descriptive study in the Netherlands. Clinical Rehabilitation. 2008; 22(12):1127-33. doi: 10.1177/0269215508095088

[13] Kadaba MP, Ramakrishnan HK, Wootten ME, Gainey J, Gorton G, Cochran GVB. Repeatability of kinematic, kinetic, and electromyographic data in normal adult gait. Journal of Orthopaedic Research. 1989; 7(6):849-60. doi: 10.1002/jor.1100070611

[14] Karimi M, Kamali M, Omar H, Mostmand J. Evaluation of gait performance of a hemipelvectomy amputation walking with a canadian prosthesis. Case Reports in Orthopedics. 2014; 2014. doi: $10.1155 / 2014 / 962980$
[15] Czerniecki JM. Rehabilitation in limb deficiency. Gait and motion analysis. Archives of Physical Medicine and Rehabilitation. 1996; 77(3):3-8. doi: 10.1016/s0003-9993(96)90236-1

[16] Radin EL, Parker HG, Pugh JW, Steinberg RS, Paul IL, Rose RM. Response of joints to impact loading-III. Journal of Biomechanics. 1973; 6(1):51-7. doi: 10.1016/0021-9290(73)90037-7

[17] Hurwitz DE, Sumner DR, Block JA. Bone density, dynamic joint loading and joint degeneration. Cells Tissues Organs. 2001; 169(3):201-9. doi: 10.1159/000047883

[18] Jaegers SMHJ, Arendzen JH, de Jongh HJ. Prosthetic gait of unilateral transfemoral amputees: A kinematic study. Archives of Physical Medicine and Rehabilitation. 1995; 76(8):736-43. doi: 10.1016/s0003-9993(95)80528-1

[19] Gitter A, Czerniecki J, Weaver K. A reassessment of center-ofmass dynamics as a determinate of the metabolic inefficiency of above-knee amputee ambulation. American Journal of Physical Medicine \& Rehabilitation. 1995; 74(5):332-8. PMID: 7576408

[20] Haberman A. Mechanical properties of dynamic energy return prosthetic feet. Ontario: Queen's University; 2008.

[21] Hall MG, Fleming HE, Dolan MJ, Millbank SFD, Paul JP. Static in situ calibration of force plates. Journal of Biomechanics. 1996; 29(5):659-65. doi: 10.1016/0021-9290(95)00109-3

[22] Keselman HJ, Othman AR, Wilcox RR, Fradette K. The new and improved two-sample t-test. Psychological Science. 2004; 15(1):47-51. doi: 10.1111/j.0963-7214.2004.01501008.x

[23] Whittle MW. Gait analysis: An introduction. London: Butterworth-Heinemann; 2014

[24] Sjödahl C, Jarnlo GB, Söderberg B, Persson BM. Kinematic and kinetic gait analysis in the sagittal plane of trans-femoral amputees before and after special gait re-education. Prosthetics and Orthotics International. 2002; 26(2):101-12. doi: $10.1080 / 03093640208726632$

[25] McNealy LL, Gard S. Effect of prosthetic ankle units on the gait of persons with bilateral trans-femoral amputations. Prosthetics and Orthotics International. 2008; 32(1):111-26. doi: $10.1080 / 02699200701847244$

[26] Sadeghi H, Allard P, Duhaime M. Muscle power compensatory physical medicine and rehabilitation. Ovid Technologies. 2001; 80(1):25-32. doi: 10.1097/00002060-200101000-00007

[27] Seroussi RE, Gitter A, Czerniecki JM, Weaver K. Mechanical work adaptations of above-knee amputee ambulation. Archives of Physical Medicine and Rehabilitation. 1996; 77(11):1209-14. doi: 10.1016/s0003-9993(96)90151-3

[28] Oatis C. Kynesiology: The mechanics and pathomechanics of human movement. Philadelphia: Lippincott Williams \& Wilkins; 2004.

[29] Isakov E, Burger H, Krajnik J, Gregoric M, Marincek C. Influence of speed on gait parameters and on symmetry in transtibial amputees. Prosthetics and Orthotics International. 1996; 20(3):153-8. PMID: 8985994 
